\title{
Revealing the state space of turbulence using machine learning
}

\author{
Jacob Page $\odot,{ }^{1,2, *}$ Michael P. Brenner, ${ }^{3,4}$ and Rich R. Kerswell $\circledast^{2}$ \\ ${ }^{1}$ School of Mathematics, University of Edinburgh, Edinburgh, EH9 3FD, United Kingdom \\ ${ }^{2}$ DAMTP, Centre for Mathematical Sciences, University of Cambridge, \\ Cambridge CB3 OWA, United Kingdom \\ ${ }^{3}$ School of Engineering and Applied Sciences, Harvard University, Cambridge, Massachusetts 02138, USA \\ ${ }^{4}$ Google Research, Mountain View, California 94043, USA
}

(Received 24 October 2020; revised 15 February 2021; accepted 22 February 2021; published 25 March 2021)

\begin{abstract}
Despite the apparent complexity of turbulent flow, identifying a simpler description of the underlying dynamical system remains a fundamental challenge. Capturing how the turbulent flow meanders among unstable states (simple invariant solutions) in phase space, as envisaged by Hopf [Commun. Pure Appl. Math. 1, 303 (1948)], using some efficient representation offers the best hope of doing this, despite the inherent difficulty in identifying these states. Here, we make a significant step toward this goal by demonstrating that deep convolutional autoencoders can identify low-dimensional representations of two-dimensional turbulence which are closely associated with the simple invariant solutions characterizing the turbulent attractor. To establish this, we develop latent Fourier analysis that decomposes the flow embedding into a set of orthogonal latent Fourier modes which decode into physically meaningful patterns resembling simple invariant solutions. The utility of this approach is highlighted by analyzing turbulent Kolmogorov flow (monochromatically forced flow on a $2 \mathrm{D}$ torus) at $\mathrm{Re}=40$, where, in between intermittent bursts, the flow resides in the neighborhood of an unstable state and is very low dimensional. Projections onto individual latent Fourier wavenumbers reveal the simple invariant solutions organizing both the quiescent and bursting dynamics in a systematic way inaccessible to previous approaches.
\end{abstract}

DOI: 10.1103/PhysRevFluids.6.034402

\section{INTRODUCTION}

Building effective low-order representations of turbulent flows is a long-standing challenge that could dramatically improve our capabilities for prediction and control. Current state-of-the-art techniques for low-order modeling typically involve constructing a set of orthogonal modes from a data set. Perhaps most well-known is principal component analysis (PCA), which produces an orthogonal basis to optimally represent the training snapshots. However, while highly interpretable, modes in the basis may have little dynamical significance individually [1], and other methods that attempt to also infer dynamics — for example, dynamic mode decomposition [2] — are ill-suited to chaotic systems like turbulence [3]. In contrast, in a dynamical systems approach, the building blocks are the simple invariant solutions which populate the chaotic attractor in a high-dimensional state space. A turbulent flow is then viewed as a long nonclosing orbit in this state space, transiting between these unstable simple invariant solutions guided by their stable and unstable manifoldsa viewpoint advocated by Hopf seven decades ago [4]. Such a viewpoint suggests that there is an efficient low-order representation of the flow rooted in the underlying simple invariant solutions.

\footnotetext{
*Corresponding author: jacob.page@ed.ac.uk
} 
Moreover, these simple invariant solutions offer valuable insight into the self-sustaining processes underpinning the turbulent dynamics due to their simple dependence on time [5-9]. With a large enough collection of simple invariant solutions, statistical predictions can then be attempted using, for example, periodic orbit theory [10,11], while continuation of the solutions along their solution curves (upward in Re) offers a plausible method to make predictions at high Reynolds numbers without simulating the fully turbulent flow.

However, attempts to implement and test these ideas are constrained by our limited ability to identify simple invariant solutions from a turbulent data set. The current state-of-the-art relies on the detection of near recurrences in a time series, which are quantified by a Euclidean distance in the state space of discretized solutions [5,11]. These guesses are then fed into a Newton-Raphson solver to determine whether the recurrent episode did indeed correspond to the shadowing of a simple invariant solution. The approach has identified large numbers of periodic orbits in canonical flows, e.g., Refs. [9,11] but crucially the overwhelming majority of solutions have been restricted to regions of the state space associated with low turbulent kinetic energy. The inability of current tools to identify simple invariant sets associated with strongly dissipative events weakens our physical understanding of the underlying physical processes and suggests that the approach will be challenging to upscale to high Re.

Many of the current limitations in the application of dynamical systems theory to turbulence can be connected to our inability to accurately parameterize the inertial manifold of solutions to the Navier-Stokes equations. The recent emergence of deep convolutional neural networks (CNNs) represents an opportunity to make progress here due to their ability to identify and extract patterns $[12,13]$. This capability can be used to generate highly efficient representations of complex data, encoded in latent ("hidden") variables within the neural network. The utility of CNNs in the study of nonlinear partial differential equations (PDEs) has been demonstrated recently in a number of canonical examples, where their accurate parameterization of the solution manifold has been exploited to successfully predict chaotic dynamics for multiple Lyapunov times [14], to estimate the dimension of chaotic attractors [15], and to design new spatial discretization schemes [16].

Using a CNN to decompose a turbulent flow into a series of recurrent spatial patterns should be contrasted to a projection onto a handcrafted orthogonal basis such as Fourier modes, where the coupling of all wavenumbers through the nonlinearity of the Navier-Stokes equation renders individual modes dynamically insignificant. A learned basis has the potential to encode and parameterize the alphabet of dynamical processes present, though at a loss of physical interpretability. Here we show how the presence of a continuous symmetry in the physical system can be exploited to perform a decomposition of embeddings of a turbulent flow in latent space. This latent Fourier analysis - analogous to a Fourier decomposition in physical space-yields a (latently) orthogonal basis of recurrent patterns that exhibit striking resemblance to simple invariant solutions of the underlying dynamical system.

\section{DIMENSIONALITY REDUCTION OF KOLMOGOROV FLOW}

We use deep CNNs to build efficient low-dimensional representations of snapshots from a computation of monochromatically forced, two-dimensional turbulence on a doubly periodic square $[0,2 \pi] \times[0,2 \pi]$. In two dimensions, the Navier-Stokes equations can be combined and written concisely in terms of the out-of-plane vorticity $\omega:=(\nabla \times \mathbf{u}) \cdot \hat{\mathbf{z}}$,

$$
\partial_{t} \omega+\mathbf{u} \cdot \nabla \omega=\frac{1}{\operatorname{Re}} \nabla^{2} \omega+(\nabla \times \mathbf{f}) \cdot \hat{\mathbf{z}}
$$

where $\mathbf{f}=\sin 4 y \hat{\mathbf{x}}$ (Kolmogorov flow [17] with the specific choice of four forcing wavelengths in the square $[11,18,19])$. There are a number of symmetries, the most important in the context of this paper being the continuous translational symmetry $\mathscr{T}_{s}: \omega(x, y) \rightarrow \omega(x+s, y)$. There is also a discrete shift-reflect symmetry $\mathscr{S}: \omega(x, y) \rightarrow-\omega(-x, y+\pi / 4)$ and a rotational symmetry $\mathscr{R}:$ 

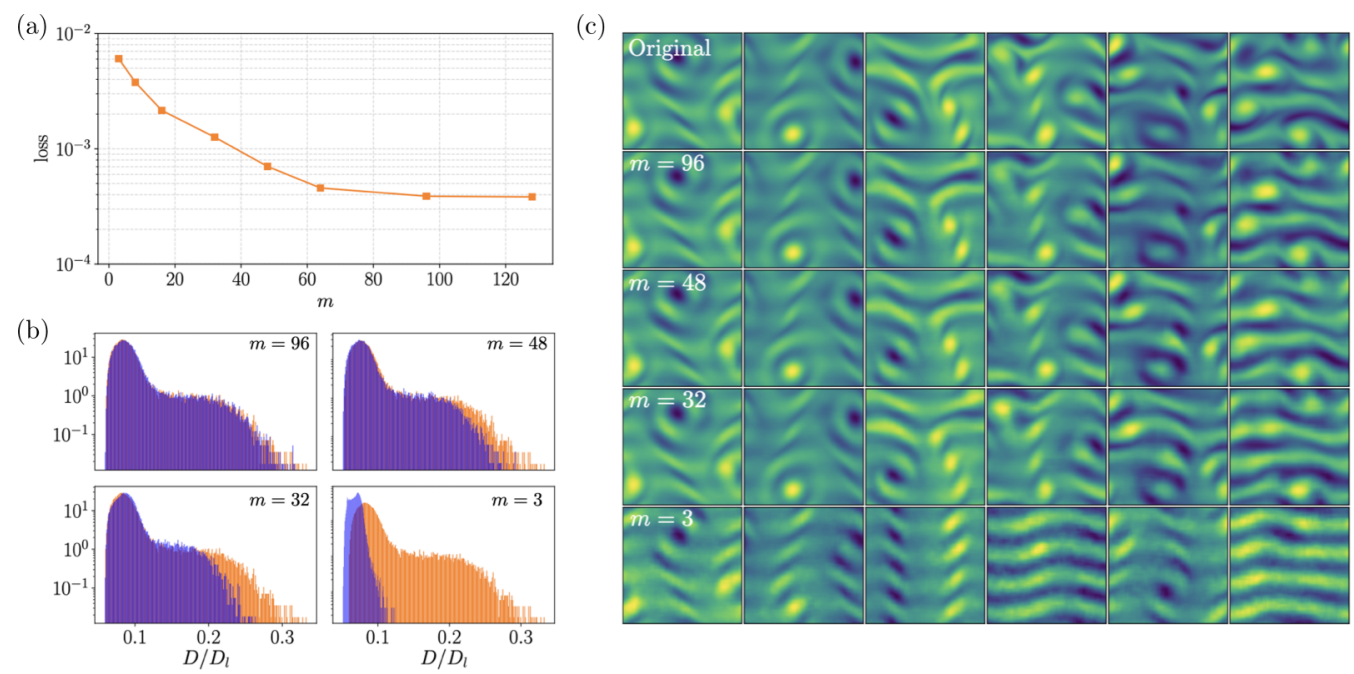

FIG. 1. Autoencoders for dimensionality reduction in Kolmogorov flow. (a) Loss $\frac{1}{\text { |data| }} \sum_{\text {data }} \|[\mathscr{D} \circ$ $\mathscr{E}](\omega)-\omega \|_{2}^{2}$ as a function of embedding dimension $m$. (b) Dissipation PDFs of autoencoded vorticity fields. The background orange is obtained from the original test data set, the overlayed blue PDFs are for $m \in\{96,48,32,3\}$ (also labeled in the figure). (c) Decodes of a set of snapshots of increasing dissipation for various embedding dimensions $m$. Snapshots are ordered left to right by dissipation, running from $D / D_{l} \approx 0.08$ to $D / D_{l} \approx 0.26$, indicating, for example, that $m=3$ captures low-dissipation episodes well but struggles to represent high-dissipation events.

$\omega(x, y) \rightarrow \omega(-x,-y)$. Throughout, we hold the Reynolds number fixed at $\mathrm{Re}=40$, where a large number of simple invariant solutions have been found [11,19].

We seek efficient low-dimensional embeddings $\{\mathscr{E}\}$ of vorticity snapshots $\{\omega\}$, which are essentially gray-scale images of dimension $N_{x} \times N_{y}=128 \times 128$. To do this, we construct deep CNNs in the form of autoencoders, which are trained to reconstruct the input snapshots with dimension reduction applied as part of the network structure. The specific architectures we use are described in detail in Appendix B, but all consist of an encoder module, $\mathscr{E}: \mathbb{R}^{128 \times 128} \rightarrow \mathbb{R}^{m}$-consisting of a series of five convolutional layers with pooling to reduce the dimension from $128^{2}$ to 128 followed by fully connected layers to further reduce the dimension to $m \leqslant 128$ (the embedding). A similar structure is used to decode the embeddings, $\mathscr{D}: \mathbb{R}^{m} \rightarrow \mathbb{R}^{128 \times 128}$ and the weights that define these functions are obtained by performing stochastic gradient descent on the loss functional $\frac{1}{\text { |data }} \sum_{\text {data }}\|[\mathscr{D} \circ \mathscr{E}](\omega)-\omega\|_{2}^{2}$.

The impact of the autoencoder dimension on the fidelity of the reconstruction $[\mathscr{D} \circ \mathscr{E}](\omega)$ is examined in Fig. 1. The loss drops monotonically with increasing $m$, with even very low-dimensional networks (e.g., $m=3$ ) displaying relatively small losses (two unrelated vorticity fields typically yield an $O(1)$ loss), which suggests that much of the underlying dynamical system may be low dimensional. Furthermore, networks with modest $m$ (e.g., see the PDF for $m=32$ in Fig. 1) retain much of the high-dissipation tail in PDFs of $D:=\left\langle(\nabla \mathbf{u})^{2}\right\rangle_{V}=\left\langle\omega^{2}\right\rangle_{V}$. This indicates a retention of smaller scales and sharp variations in $\omega$ under dimensionality reduction, in contrast to standard techniques like PCA. Even at $m=3$, the accurate reproductions of low dissipation events (see the snapshots in Fig. 1) contain the full spectrum of Fourier modes.

To examine how these networks can reduce the dimensionality of the data while retaining a broad spectrum of lengthscales, we describe a method for decomposing the latent representations of vortical snapshots into a finite set of recurrent patterns which can be visualized individually. 


\section{LATENT FOURIER ANALYSIS}

The continuous translational symmetry in the governing Eq. (1) and boundary conditions provides a mechanism to decompose the latent embeddings of the vorticity fields into recurrent patterns that reveal the structure of the latent space. This decomposition is analogous to a Fourier transform in physical space, $\omega(x, y, t)=\sum_{k \in \mathbb{Z}} \hat{\omega}_{k}(y, t) \exp (i k x)$. However, the autoencoder representations encode the horizontal position $x$ in an unknown way. To perform a similar decomposition for embeddings, we first must construct an operator that can map between an embedding of a snapshot and an embedding of a shifted version of the same field: $\mathbf{T}_{\alpha} \mathscr{E}(\omega)=\mathscr{E}\left(\mathscr{T}_{\alpha} \omega\right)$, where the fixed shift $\alpha \in$ $(0,2 \pi)$ is a design choice. To generate $\mathbf{T}_{\alpha}$, a matrix of embeddings, $\mathbf{E}:=\left[\begin{array}{lll}\mathscr{E}\left(\omega_{1}\right) & \cdots & \mathscr{E}\left(\omega_{N}\right)\end{array}\right]$ is assembled along with another data matrix built from embeddings of the same vorticity fields shifted by $\alpha$ in $x, \mathbf{E}^{\prime}:=\left[\begin{array}{lll}\mathscr{E}\left(\mathscr{T}_{\alpha} \omega_{1}\right) & \cdots & \mathscr{E}\left(\mathscr{T}_{\alpha} \omega_{N}\right)\end{array}\right]$. An approximate shift operator is then determined from a least-squares fit over the test set, $\mathbf{T}_{\alpha}=\mathbf{E}^{\prime} \mathbf{E}^{+}$, where $\mathbf{E}^{+}$is the Moore-Penrose pseudoinverse of $\mathbf{E}$. This algorithm is well-known in the fluid dynamics community, where it is typically applied to temporally spaced flow snapshots to extract dynamic modes with an exponential dependence on time $[2,3,20]$.

This procedure applied to the vorticity field itself would result in a numerical approximation to a Fourier transform through the eigenvalues and eigenvectors of $\mathbf{T}_{\alpha}$, with a maximum resolved wavenumber set by the Nyquist condition, $k_{\max }=\pi / \alpha$. For the embeddings, $\mathscr{E}$, the value of $\alpha$ sets a maximum latent wavenumber that can be resolved, $l_{\max }$. As we will show below, the required latent resolution is considerably coarser than the smallest scales generated by the governing PDE Eq. (1).

To see the connection to a standard Fourier transform, consider a discrete shift $\alpha=2 \pi / n$, with $n \in \mathbb{N}$. By design, $\mathbf{T}_{\alpha}^{n} \mathscr{E}(\omega)=\mathscr{E}(\omega)$, so the eigenvalues of $\mathbf{T}_{\alpha}$ are $\Lambda_{j}=\exp \left(2 \pi i l_{j} / n\right)$, with $l_{j} \in \mathbb{Z}$. We assume that the value of $\alpha$ has been chosen small enough so no $l_{j}$ are found beyond a maximum $\left(l_{\max }\right)$. With this, approximations to continuous shifts $s$ of an embedding $\mathscr{E}(\omega)$ are

$$
\begin{aligned}
\mathscr{E}\left(\mathscr{T}_{s} \omega\right) & =\sum_{l}\left(\sum_{j=0}^{d(l)-1} \mathcal{P}_{j}^{l}(\mathscr{E}(\omega))\right) e^{i l s} \\
& :=\sum_{l}\left(\sum_{j=0}^{d(l)-1}\left[\left(\xi_{j}^{\dagger(l)}\right)^{H} \mathscr{E}(\omega)\right] \boldsymbol{\xi}_{j}^{(l)}\right) e^{i l s}
\end{aligned}
$$

Here $l$ is the latent wavenumber and the operator $\mathcal{P}_{j}^{l}$ is a projector in direction $j$ within the eigenspace of wavenumber $l$, which has geometric multiplicity $d(l)$. Unlike physical Fourier modes, the latent wavenumbers are degenerate: $\xi_{j}^{(l)}$ being the $(j+1)$ th eigenvector with wavenumber $l$. Equation (2) assumes that some biorthogonal basis $\xi_{j}^{\dagger(l)}$ has been constructed; a specific choice is discussed further below.

The number of required latent wavenumbers and their degeneracy provides insight into the nonlinear interactions in physical space. Each latent Fourier mode of wavenumber $l$ can be decoded into a $2 \pi / l$-periodic pattern which has a physical Fourier decomposition projecting onto wavenumbers $k_{q}=q l, q \in \mathbb{N}$ (see the example in the schematic of Fig. 2). Significantly, these recurrent patterns represent pathways through physical Fourier wave numbers which are selected by the dynamics.

The maximum required latent wavenumber, $l_{\max }$, is determined empirically via the computation of a set of several shift operators in latent space, $\mathbf{T}_{\alpha}$, for various network dimensions $m$ and shifts $\alpha$. The eigenvalue spectra of some of these operators are reported in Fig. 3 in timestepper form. Latent wavenumbers can be extracted via $l=\ln \Lambda /(i \alpha)$. At fixed $\alpha$, the number of required latent wavenumbers saturates at $l=3$ beyond $m=32$. At fixed $m$, no further latent wavenumbers are recovered for shifts $\alpha<2 \pi / 6$. Therefore, any shift $\alpha<2 \pi / 6$ is sufficient to perform a latent Fourier transform without any aliasing issues. Moreover, the truncation suggests that the learned representations can be decomposed into a set of recurrent patterns which are at most $2 \pi / 3$ periodic. 


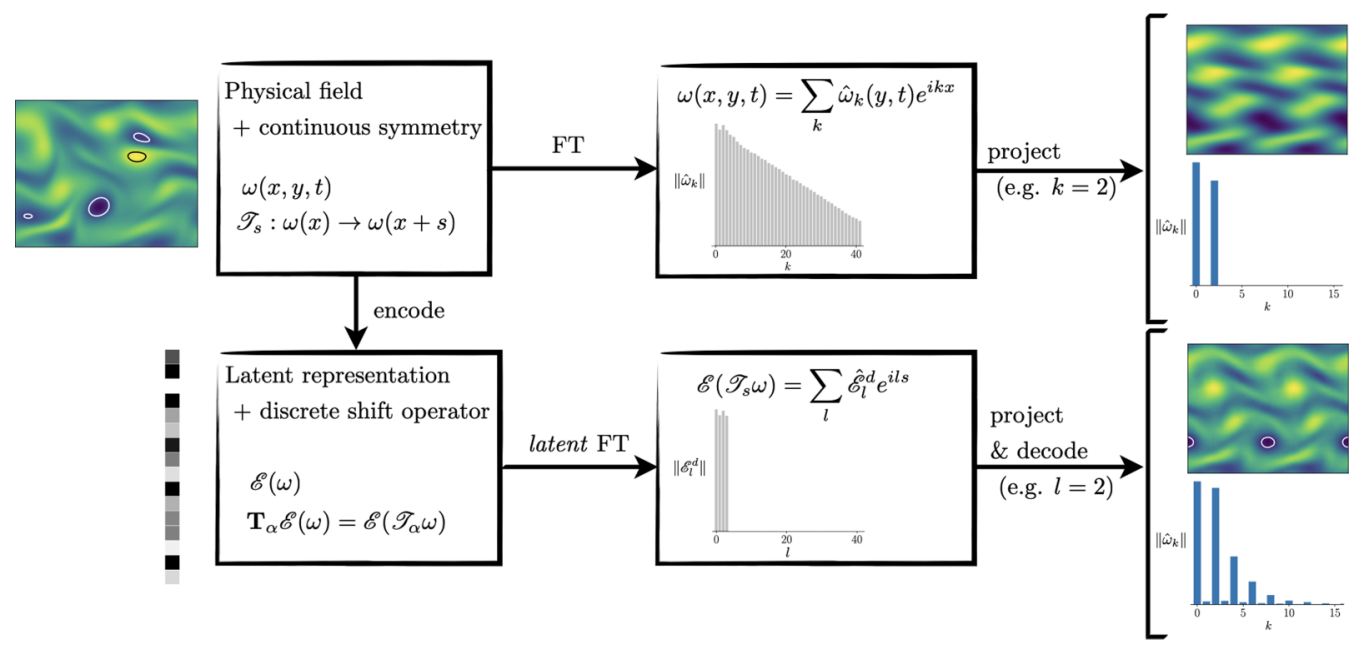

FIG. 2. An overview of the approach to performing latent Fourier decompositions on (latent) representations of physical systems with a continuous symmetry. A latent Fourier decomposition is performed by constructing an operator to map between embeddings of shifted versions of the same snapshot. Note that only four latent wavenumbers, $\{0,1,2,3\}$, are required for the monochromatically forced turbulence considered here. In the project and decode step (bottom right), the projection within the $l=0$ subspace is also included (see the discussion in the text). For comparison, the projection onto $k=0$ is also included in the projection onto the physical Fourier modes.

(a)

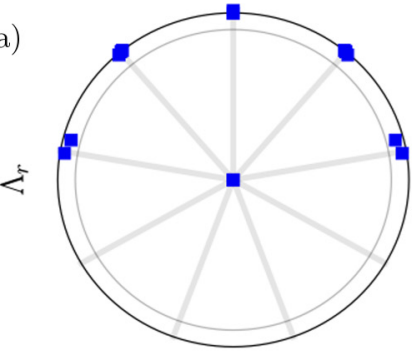

$\Lambda_{i}$

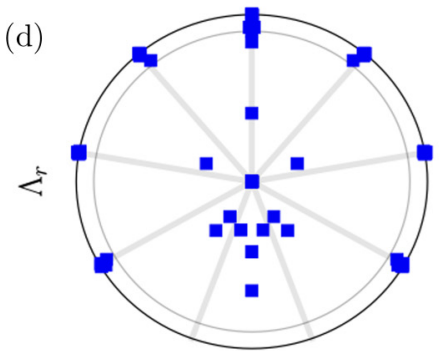

$\Lambda_{i}$ (b)

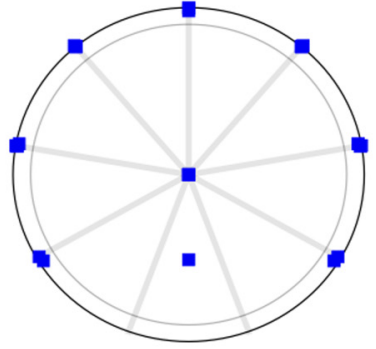

$\Lambda_{i}$

(e)

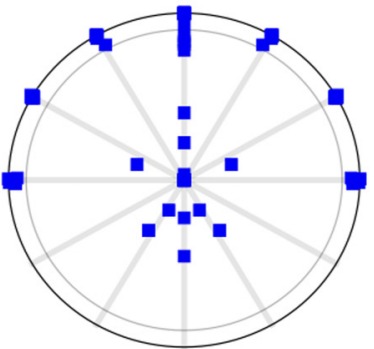

$\Lambda_{i}$ (c)

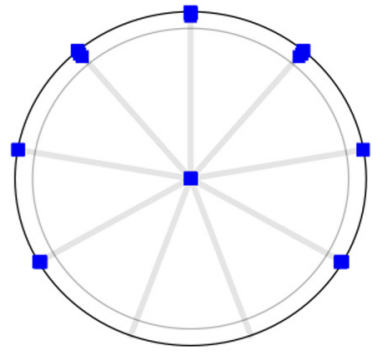

$\Lambda_{i}$

(f)

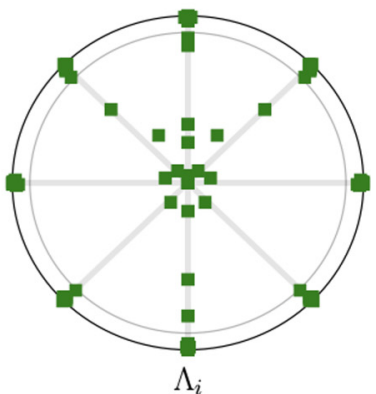

FIG. 3. Eigenvalue spectra of the latent symmetry operators for various embedding sizes $m$ and shifts $\alpha$. Panels (a) -(c) have fixed $\alpha=2 \pi / 9$ and varying $m$ : (a) $m=16$, (b) $m=32$, (c) $m=64$. Panels (d) and (e) have fixed $m=96$ and varying $\alpha$ : (d) $\alpha=2 \pi / 9$, (e) $\alpha=2 \pi / 12$. Finally, panel (f) displays the eigenvalues of a numerical approximation to the vertical shift-reflect operator, $\mathbf{S}$ at $m=96$. In all cases, the outer black circle is $|\Lambda|=1$, the grey interior circle is $|\Lambda|=0.9$. The rays from the origin identify the relevant roots of unity. 


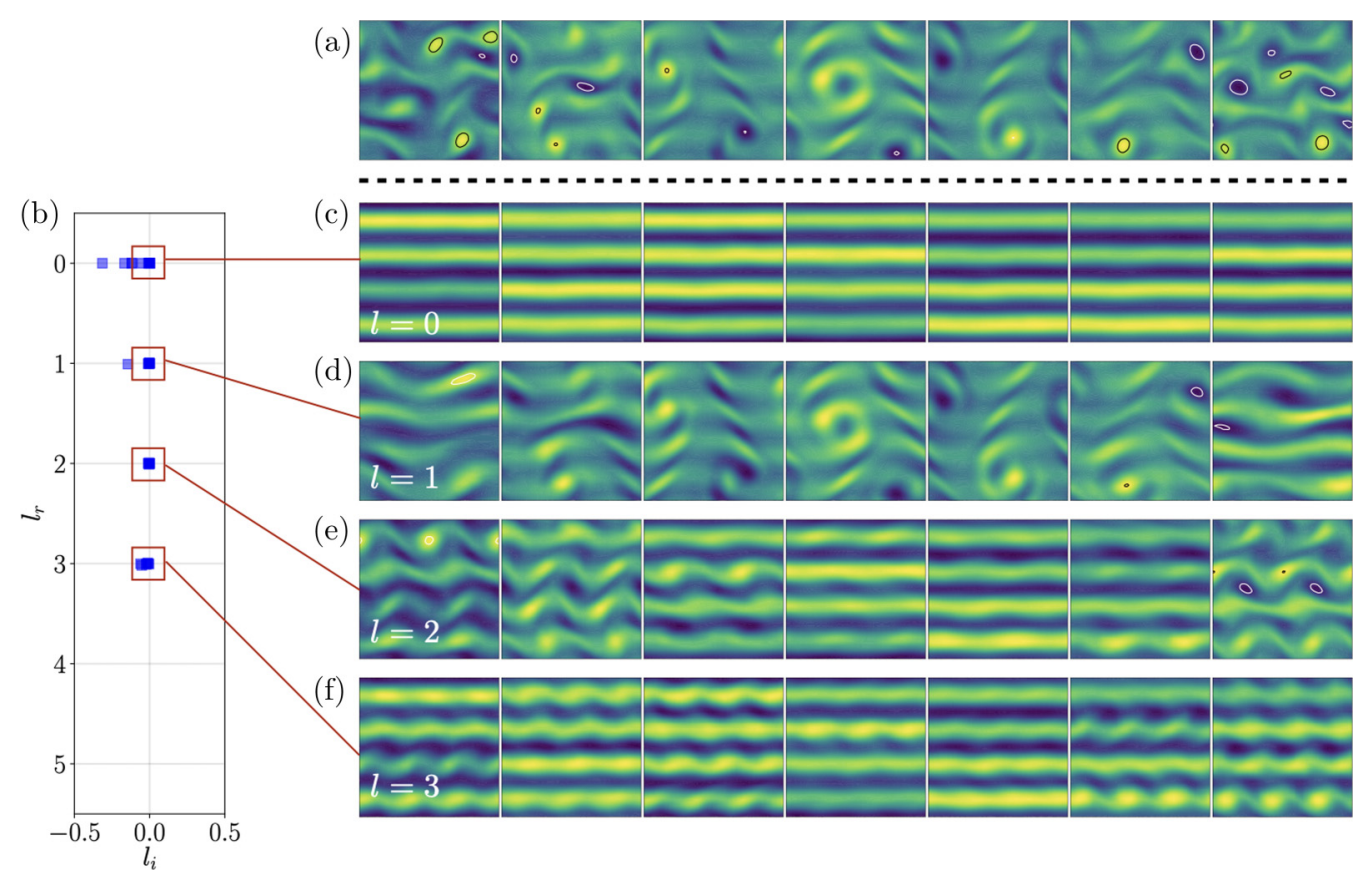

FIG. 4. Eigenvalue spectra of a latent symmetry operator and decodes of the projection onto individual eigenspaces for example snapshots. (a) Seven example snapshots of vorticity, $\omega$. (b) Eigenvalue spectrum of $\mathbf{T}_{\alpha}$ on the $m=96$ autoencoder with $\alpha=2 \pi / 9$. Note that only half the spectrum is shown. (c)-(f) Decodes of the snapshots in (a) after their embeddings are projected onto individual latent Fourier eigenspaces [latent wavenumber $l$ is identified in each row and by the red line from the eigenvalue spectrum (b)]. Note that the $l=0$ subspace is always included for decodes of latent wavenumbers $l>0$.

The energy in all higher physical wavenumbers, $k>3$, is assigned during the decode of this coarse set of features in the latent space (e.g., $k=5$ can only be encoded into $l=1$ and $k=8$ into $l=2$ ).

An example eigenvalue spectrum for the $m=96$ network is reported in Fig. 4 in exponential form; the (degenerate) eigenvalues lying approximately on $l \in\{0,1,2,3\}$. Some example $2 \pi / l$ periodic physical patterns associated with a particular value of $l$ are displayed alongside the spectrum. These images were generated by projecting the embedding of a snapshot onto the relevant eigenspace and decoding the result. Note that, in contrast to a standard Fourier transform, the $l=0$ contribution must always be included for the decode operation to yield a physical field. Projections onto the $l=0$ subspace decode to horizontal stripes of vorticity which align with the (curl of) the forcing in Eq. (1); the vorticity amplitude of each stripe is distorted by the $l>0$ modes into vortical features. In this way, much of the $y$ dependence in the final decoded snapshot is controlled by the $l=0$ subspace.

The decodes in Fig. 4 for $l>0$ have the expected periodicity, $\mathscr{T}_{2 \pi / l}\left[\mathscr{D}\left(\sum_{j} \mathcal{P}_{j}^{l}(\mathscr{E})\right)\right]=$ $\mathscr{D}\left(\sum_{j} \mathcal{P}_{j}^{l}(\mathscr{E})\right)$. In contrast to a projection onto individual Fourier modes in physical space, the projection onto individual latent wavenumbers produces patterns with vortical features that can be clearly identified in the original snapshots. The wide range of features observed in decodes of individual latent wavenumbers is possible due to the degeneracy of the eigenspaces and is discussed further in the next section.

Finally, it is worth remarking that a similar approach to that outlined above can be applied to discrete symmetries. For example, a linear operator can be sought which performs the shift-reflect 


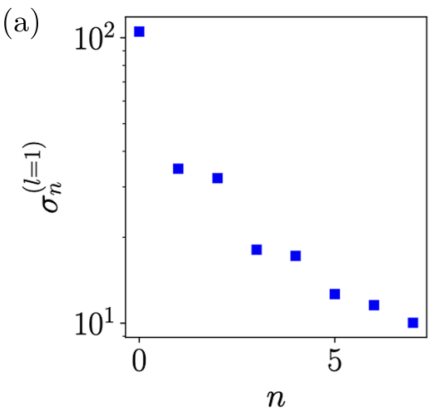

(b)

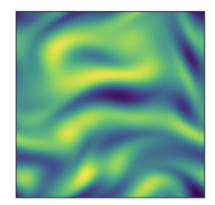

(c)
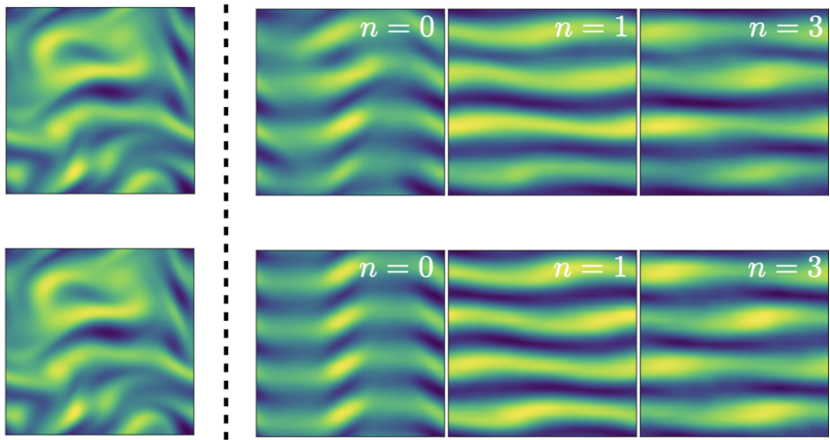

FIG. 5. Visualization of the degenerate eigenspace $l=1$ via decodes of projections onto its PCA modes for an example snapshot. (a) Singular values from the $l=1$ subspace. (b) An example vortical snapshot (to the left of the dashed black line) and the decode of the projection of its embedding onto individual PCA modes $\left\{\boldsymbol{u}_{n}^{(1)}\right\}$ within the $l=1$ subspace; note the $l=0$ projection is also included [see Eq. (6)]. (c) As (b) but only the first PCA mode from the $l=0$ subspace is used [see Eq. (7)].

operation in latent space:

$$
\mathbf{S} \mathscr{E}(\omega)=\mathscr{E}(\mathscr{S} \omega)
$$

Eight shift-reflect operations return the vorticity field to its original configuration, $\mathscr{S}^{8} \omega=\omega$, so the eigenvalues of $\mathbf{S}$ are the eighth roots of unity. Numerical results reported in Fig. 3 indicate that this structure is preserved in the latent space of our autoencoders. While the resulting decomposition of embeddings can be used to extract or identify vortical patterns which are invariant under certain numbers of shift-reflects, the minimum vertical lengthscale of the associated recurrent patterns are restricted to $L_{\min }<\pi / 4$ by the Nyquist criterion. As a result, smaller scales are hidden within the degenerate eigenspaces and cannot be revealed by reducing the vertical shift, which is fixed by the discrete symmetry.

\section{CONNECTIONS TO SIMPLE INVARIANT SOLUTIONS}

The degenerate set of recurrent patterns encoded within each latent eigenspace of the $x$-shift operator $\mathbf{T}_{\alpha}$ can be revealed by an appropriate choice of basis to define the projectors $\mathcal{P}_{j}^{l}$ in Eq. (2). We have found PCA within each eigenspace to be robust for this purpose. To perform the PCA, we first construct an arbitrary biorthogonal basis from the numerically computed left and right eigenvectors of $\mathbf{T}_{\alpha}$,

$$
\begin{gathered}
\mathbf{\Xi}_{l}=\mathbf{V}_{l} \mathbf{R}^{-1}, \\
\mathbf{\Xi}_{l}^{\dagger}=\left(\mathbf{Q}^{-1} \mathbf{W}_{l}^{H}\right)^{H},
\end{gathered}
$$

where the columns of $\mathbf{V}_{l}$ are the numerically computed right eigenvectors of $\mathbf{T}_{\alpha}$ corresponding to wavenumber $l$; the columns of $\mathbf{W}_{l}$ are the left eigenvectors. The matrices $\mathbf{Q}$ and $\mathbf{R}$ are the $\mathrm{QR}$ decomposition of $\mathbf{W}_{l}^{H} \mathbf{V}_{l}$. The columns of $\boldsymbol{\Xi}$ and $\boldsymbol{\Xi}^{\dagger}$ form a biorthogonal basis, $\left(\boldsymbol{\xi}_{i}^{\dagger(l)}\right)^{H} \boldsymbol{\xi}_{j}^{(l)}=\delta_{i j}$. We then compute the projections of our test set of embeddings within each eigenspace, and perform PCA on the resulting data matrix to form an orthogonal basis ordered by 'energy'.

The decomposition within $l=0$, while not particularly informative on its own, is most useful for visualizing other eigenspaces, because a projection onto the leading PCA mode in $l=0, \boldsymbol{u}_{0}^{(l=0)}$, decodes a vorticity field resembling the laminar parallel flow solution. This field is invariant under all symmetry operations, allowing symmetries within the $l>0$ eigenspaces to be identified. 
A singular value decomposition within the $l=1$ subspace is reported in Fig. 5 and reveals the presence of a large-amplitude leading mode with higher order modes appearing in pairs at lower energies. To visualize individual modes from within the $l=1$ subspace, we must also choose some contribution from the $l=0$ subspace. In Fig. 5, we show decodes of $l=1$ modes using both the full $l=0$ subspace,

$$
\omega_{n}^{(1)}=\mathscr{D}\left(\sum_{j=0}^{d(0)-1} \mathcal{P}_{j}^{0}+\left[\mathcal{P}_{n}^{1}+\text { c.c. }\right]\right),
$$

and also the leading $l=0$ mode only,

$$
\bar{\omega}_{n}^{(1)}=\mathscr{D}\left(\mathcal{P}_{0}^{0}+\left[\mathcal{P}_{n}^{1}+\text { c.c. }\right]\right) .
$$

where, e.g., $\mathcal{P}_{j}^{0}=\mathcal{P}_{j}^{0}(\mathscr{E}(\omega))$. As described above, the use of $\mathcal{P}_{0}^{0}$ alone removes much of the $y$ dependence when visualizing $l=1$ modes from projections of arbitrary snapshots due to the high degree of symmetry associated with $\boldsymbol{u}_{0}^{(0)}$.

Decoding projections $\bar{\omega}_{0}^{(1)}$ with Eq. (7) reveal a structure that is symmetric under rotation and shift-reflects, $\bar{\omega}_{0}^{(1)}=\mathscr{R} \bar{\omega}_{0}^{(1)}, \bar{\omega}_{0}^{(1)}=\mathscr{S}^{m} \bar{\omega}_{0}^{(1)}$ (see Fig. 5). Higher order PCA modes within $l=1$ are also reported in Fig. 5 and also have a large degree of discrete symmetry. For example, the slanted vortical structures associated with mode $\boldsymbol{u}_{1}^{(1)}$ are symmetric under rotation and whole-wavelength shifts, $\bar{\omega}_{0}^{(1)}=\mathscr{R} \bar{\omega}_{0}^{(1)}, \bar{\omega}_{0}^{(1)}=\mathscr{S}^{2 m} \bar{\omega}_{0}^{(1)}$. The projection onto $\boldsymbol{u}_{2}^{(1)}$ (not shown) is simply the shiftreflect of $\boldsymbol{u}_{1}^{(1)}, \boldsymbol{u}_{2}^{(1)}=\mathscr{S}^{2 m+1} \boldsymbol{u}_{1}^{(1)}$. The same set of symmetries hold for the vortex blobs obtained when decoding projections onto $\boldsymbol{u}_{3}^{(1)}$ and $\boldsymbol{u}_{4}^{(1)}$.

Strikingly, the highly symmetric, large-amplitude primary PCA mode in the $l=1$ subspace, $\boldsymbol{u}_{0}^{(1)}$, closely resembles the equilibrium born in the continuous-symmetry-breaking bifurcation off the laminar base state at low $\operatorname{Re} \approx 10[11,21]$. We explore this connection in Fig. 6, where we show that decodes of projections onto $\boldsymbol{u}_{0}^{(0)}$ and $\boldsymbol{u}_{0}^{(1)}$ can be used to reconstruct this structure over a range of $\mathrm{Re}$, despite the fact that the training was conducted at fixed $\operatorname{Re}=40$. As the solution branch is traversed, the amplitude of the projection of the embedding onto $\boldsymbol{u}_{0}^{(1)}$ is increased. In the vorticity field, this corresponds to both a strengthening and tilting of the vorticity bands.

While it is surprising that this nontrivial, $2 \pi$-periodic equilibrium should form the backbone of the latent representations - neither it nor the laminar solution are seen explicitly during training - it is intuitive as further simple invariant solutions and the emergence of chaotic dynamics appear in bifurcations from this state. It's worth emphasizing that this structure is associated with a single latent wavenumber, $l=1$, in contrast to its physical Fourier transform which projects onto all physical wavenumbers.

The full structure of the state space of vorticity fields can be concisely visualized by first projecting embeddings of the test data set onto the latent Fourier modes,

$$
\psi(\omega):=\left(\begin{array}{c}
\boldsymbol{u}_{0}^{0 H} \mathscr{E}(\omega) \\
\boldsymbol{u}_{1}^{0 H} \mathscr{E}(\omega) \\
\vdots \\
\left|\boldsymbol{u}_{0}^{1 H} \mathscr{E}(\omega)\right| \\
\vdots \\
\left|\boldsymbol{u}_{0}^{2 H} \mathscr{E}(\omega)\right| \\
\vdots
\end{array}\right),
$$

where the first five PCA modes of each eigenspace are included, and taking the absolute value of projections onto PCA modes from eigenspaces $l \geqslant 1$ removes any dependence on the relative streamwise location of the recurrent patterns. A two-dimensional visualization is then generated by supplying this observable as input to the t-SNE algorithm [22]. The output of this pro- 
(a)
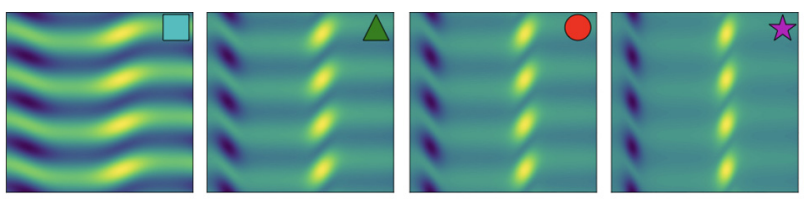

(b)
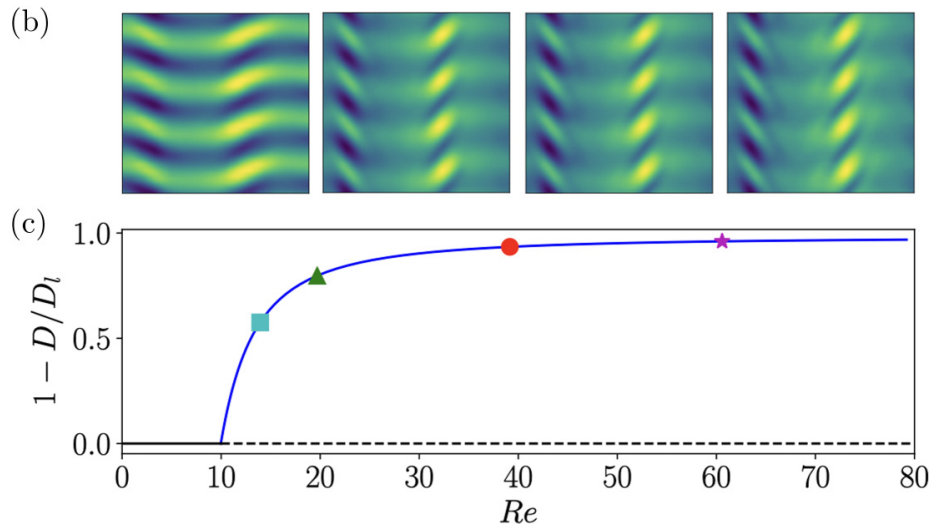

FIG. 6. The primary bifurcation of Kolmogorov flow and its connection to the primary latent Fourier mode within the $l=1$ subspace. (a) Equilibrium states at $\operatorname{Re} \approx\{14,20,40,60\}$ from the solution branch which bifurcates from the basic laminar state at $\operatorname{Re} \approx 9.97$. (b) Decode of the projection of the embeddings of the equilibria in (a) onto the first PCA modes within the $l=0$ and $l=1$ subspaces. (c) Amplitude (measured by dissipation) of this equilibrium as a function of Reynolds number (blue line, the laminar solution sits on $1-D / D_{l}=0$ ) with the example states shown above identified with symbols [see corresponding symbols in in the panels of (a)]. It should be emphasized that training has been conducted at fixed $\mathrm{Re}=40$, and that all of the training snapshots are from within the turbulent attractor and do not feature this simple equilibrium.

cedure is reported in Fig. 7 and shows a large octagon consisting of mainly low-dissipation embeddings and a detached high-dissipation cluster. Typically, the low-dissipation events require only the $l=1$ subspace, while the rarer, high-dissipation or bursting snapshots have significant projections onto the $l=2$ and $l=3$ eigenspaces. This makes it clear that there is only a single class of bursting event here. More complicated dynamics at higher Re would be reflected here by multiple, distinct projections and the necessity for higher latent wavenumber spaces.

Decoding example points from within the low-dissipation cluster reveals that its center contains snapshots that are visually similar to the first equilibrium-compare the middle flow field in Fig. 5 to Fig. 6-while embeddings of fields with pairs of opposite-sign vortices are situated toward the edges. The appearance of vortices which break the shift-reflect symmetry are indicative of secondary instabilities of the first equilibrium [21]. The eight sectors of the octagon-like cluster correspond to latent representations of the same recurrent patterns shift-reflected in the vertical direction. This effect is visualized in Fig. 7 by the square symbols in the cluster, which are eight copies of the embedding of the same vorticity field. This simple representation of the low-dissipation dynamics is retained in all autoencoders (even $m=3$, not shown). Low- $m$ networks do not build representations of the more complex bursting behavior (see Fig. 1).

The extraction of a known equilibrium from the embeddings, and the demonstration that the flow spends much of its time nearby in phase space, highlights the advantages of autoencoders as tools for generating low-dimensional representations which can be connected to the underlying dynamics. More significantly, the latent Fourier decompositions also allow us to efficiently find many simple invariant solutions of the governing Eq. (1) in parts of phase space-the bursting events-where current methods struggle, which we now describe. 


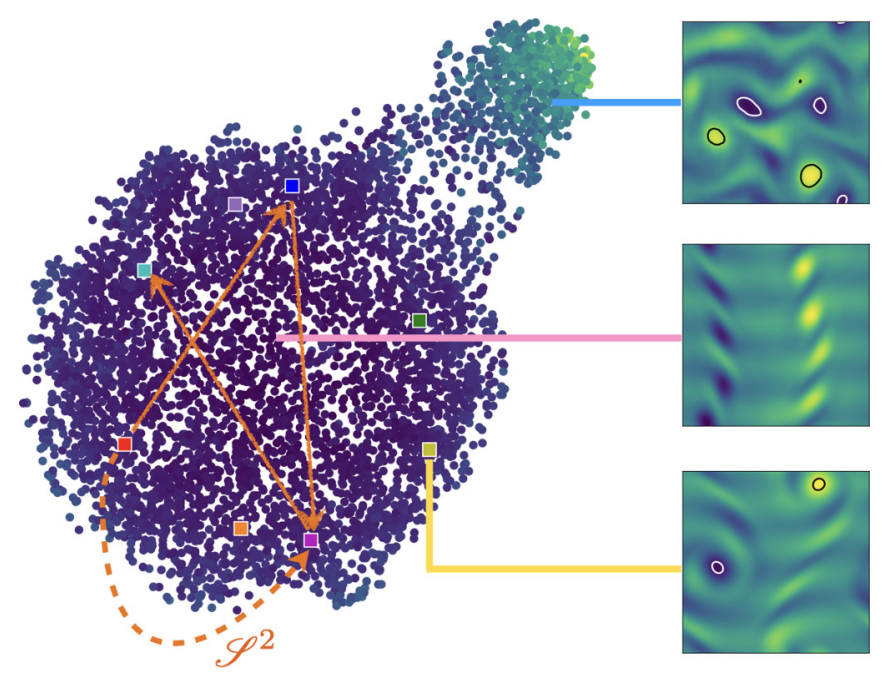

FIG. 7. Two-dimensional t-SNE of embeddings of 5000 vorticity snapshots in the $m=96$ network. The figure was created by computing projections of the embeddings onto the five leading PCA modes within each degenerate eigenspace $l \in\{0,1,2,3\}$. Translational dependence of the features was then removed by taking the absolute value of these projections for $l>0$ before the t-SNE algorithm [22] was applied. The data points are colored by their dissipation values which run between $D / D_{l}=0.06$ (dark blue) and $D / D_{l}=0.33$ (yellow). Decodes of example snapshots are shown on the right. The eight colored squares represent the embeddings of the same vorticity snapshot (see the decode of the yellow square at bottom right) with the shift-reflect operation repeatedly applied. The orange arrows indicate how this field is moved between sectors of the low-dissipation octagon under applications of a shift-reflect operation; the dashed arrow labeled $\mathscr{S}^{2}$ corresponds to a full wavelength shift in $y$.

A long time series from a turbulent computation is examined in Fig. 8, visualized both in terms of dissipation and also the magnitude of the projection of the flow embeddings onto certain latent Fourier modes. The bursting events could be classified as sections of the time series where the dissipation rate exceeds some threshold; for example, $D / D_{l}>0.15 \mathrm{might}$ be sensible here. The latent Fourier projections offer an alternative view on the bursting in terms of a distance from the simple equilibrium described above, which is central to the low-dissipation dynamics (see Fig. 7). The solid blue line in Fig. 8 shows the projection onto the latent Fourier mode which encodes this structure, $\mathcal{P}_{0}^{1}$. As expected, this equilibrium is dominant in the embedding for the low- $D$ dynamics but becomes insignificant in the (high- $D$ ) bursting events. Bursting also exhibits a dramatic increase in importance of the $l=2$ eigenspace, as well as the other modes from within $l=1$. There is also a significant projection onto $l=3$ (not shown).

Motivated by the prominent role of the $l=2$ eigenspace in the bursting, we explored how well the embedding is capturing the simple invariant solutions present in this part of phase space by supplying the decode of embeddings projected onto this space,

$$
\omega^{(2)}=\mathscr{D}\left(\sum_{j=0}^{d(0)-1} \mathcal{P}_{j}^{0}+\left[\sum_{j=0}^{d(2)-1} \mathcal{P}_{j}^{2}+\text { c.c. }\right]\right),
$$

as initial guesses in a Newton-GMRES solver searching for equilibria and traveling waves (see Appendix C). Some examples of the recurrent patterns associated with this decode were included earlier in Fig. 4; these decodes have symmetry under half domain shifts $\mathscr{T}_{\pi} \omega^{(2)}=\omega^{(2)}$. This translational symmetry matches that found in the equilibrium $E_{13}$ which was the only solution found to be important in the bursting dynamics in Ref. [19]. 

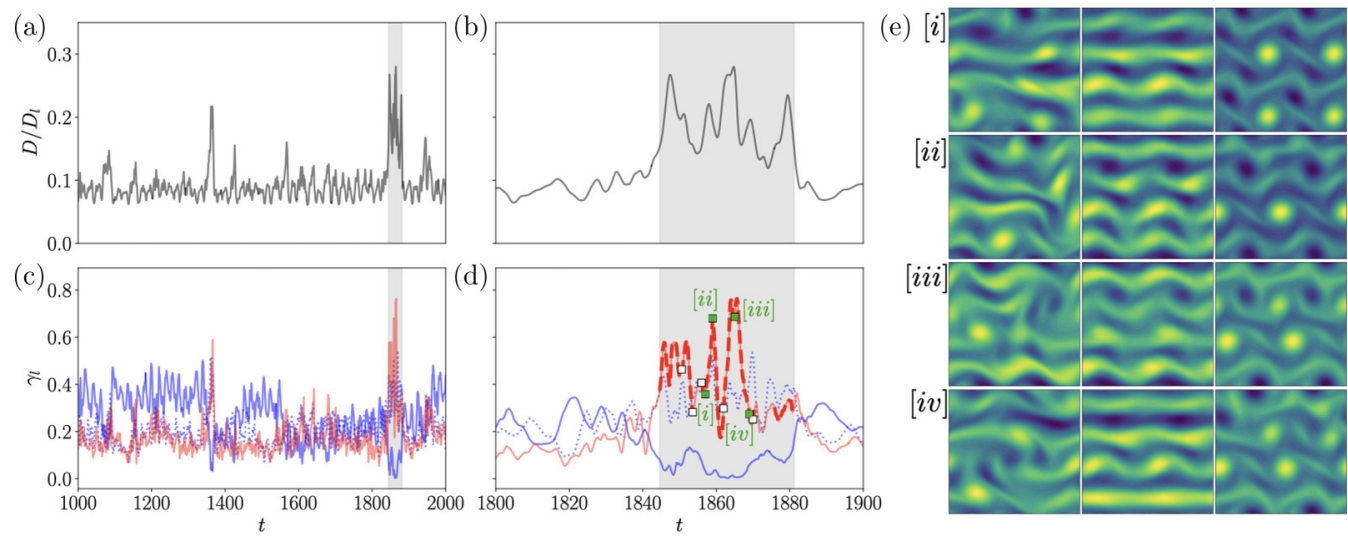

FIG. 8. Bursting episodes: Their recurrent patterns and associated exact coherent structures. (a), (b) Dissipation and (c), (d) projections onto latent wavenumbers $l=1$ and $l=2$ for a long turbulent signal [panels (b) and (d) show zoomed-in regions identified with gray shading). In (c), (d), the solid blue line is the projection onto the $l=1$ mode corresponding to the primary bifurcation in the flow, $\gamma_{1}:=\left\|\mathcal{P}_{0}^{1}(\mathscr{E}(\omega))\right\|^{2}$ (see text), the dashed blue line is the projection onto the rest of the modes in the $l=1$ eigenspace, $\gamma_{1+}:=$ $\left\|\sum_{j=1}^{d(1)-1} \mathcal{P}_{j}^{1}(\mathscr{E}(\omega))\right\|^{2}$; the solid red line is the projection onto the full $l=2$ eigenspace giving $\gamma_{2}$. In panel (d), the dashed red line overlaying the $l=2$ curve identifies regions where $D / D_{l}>0.15$. Square markers indicate where equilibria and traveling waves corresponding to the $l=2$ recurrent patterns were found; the labels [i]-[iv] next to the green markers coincide with the equilibria reported in panel (e). In panel (e), we show snapshots (left) extracted at the green markers [i]-[iv] in (d), the decode of the projection onto the $l=2$ eigenspace (middle, note this includes the $l=0$ contribution) and the converged equilibria or traveling wave (right).

By constructing guesses via Eq. (9), we have been able to converge a large number of equilibria and traveling waves directly from the bursting snapshots themselves, as well as rediscovering $E_{13}$. From an analysis of $\sim 20$ bursts within a time series of length $t \sim 4000$, we have found over 25 unique solutions, usually finding at least one simple invariant solution per burst. All of our solutions have high dissipations, with the majority having values $D / D_{l}>0.2$. We include some of these solutions in Fig. 8 alongside the original snapshots and the initial guess generated via Eq. (9). The signature of the converged solution can often be seen in the $l=2$ recurrent pattern, which exhibits vortical features also found in the original snapshot. The fact that the solutions found seem positioned at extremes of the dissipation signal—see the middle lower plot in Fig. 8-is fully consistent with the picture of the turbulent trajectory bouncing between the neighborhoods of these unstable solutions in phase space. The utility of the method is that it can identify simple invariant solutions which are actually transiently visited by the dynamics in high-dimensional bursts, which has not been possible using previous approaches. Moreover, some of our solutions are qualitatively different from any that have been converged before [11,19], for example, note the vorticity snapshots dominated by dipole structures in Fig. 10 in Appendix C.

\section{CONCLUSION}

In this paper, we have used deep convolutional autoencoders to construct efficient lowdimensional representations of monochromatically forced, two-dimensional turbulence at $\operatorname{Re}=40$. The networks are highly effective at identifying recurrent spatial patterns in the vorticity fieldcommon wavenumber pathways in a Fourier representation - in striking contrast with standard dimensionality reduction techniques. By exploiting a continuous symmetry, we have developed an interpretable latent Fourier decomposition of the embeddings: the latent Fourier modes can be decoded into physically meaningful fields. This has allowed us to reveal the structure of state 
space underlying the dynamics. One equilibrium (the primary bifurcation) dominates the quiescent low-dissipation dynamics while one grouping of simple invariant solutions organize a single type of high dissipation bursting event which occurs intermittently. The success of latent Fourier analysis in identifying dynamically important solutions of high dissipation for the bursting episodes is particularly noteworthy as previous methods $[11,19]$ have struggled to do this. Going forward, these solutions present a way of charting the bursting dynamics, as latent Fourier decompositions provide us with a natural metric for measuring which solution a turbulent orbit is nearest to. Moreover, latent Fourier analysis also allows us to efficiently find large numbers of periodic orbits, including those in previously unreachable parts of the state space (see the example in Appendix C), than has been previously possible [23].

The results presented here clearly show that harnessing machine-learning techniques to allow the building blocks of a flow representation to design themselves based on the flow dynamics is a significant step forward. The blocks which emerge are the principal spatial patterns or coherent structures observed in the flow and, intriguingly, can accurately capture simple invariant solutions embedded in the turbulent attractor without the solutions ever being realized precisely. This opens up the possibility of an easily automated, direct approach for both identifying when the flow is in the neighborhood of a state in phase space and evaluating the probability of being there. Turbulent statistics could then be predicted through a weighted sum over relevant states, for example, in the spirit of periodic orbit theory [24,25]. However, in the immediate future, the natural next step is to apply these techniques at much higher Reynolds numbers and in three dimensions. In these extensions, assessing how much data is needed to power this approach will also be an important consideration.

\section{ACKNOWLEDGMENTS}

J.P. acknowledges support from the Sultan Qaboos Fellowship at Corpus Christi College, University of Cambridge. M.P.B. acknowledges support from the Simons Foundation and also from NSF Division of Mathematical Sciences Grant No. DMS1715477.

\section{APPENDIX A: DATA}

Training data are generated by solving Eq. (1) at fixed $\mathrm{Re}=40$. For spatial discretization, we apply a two-dimensional Fourier transform at a resolution $N_{x} \times N_{y}=128 \times 128$; de-aliasing is applied according to the $2 / 3$ rule. For timestepping, an implicit Crank-Nicholson scheme is employed for the diffusion term and Heun's method is used for the nonlinear advection terms. For further details, see Refs. [11,21].

The training data set is constructed from 1000 independent trajectories each consisting of 100 snapshots separated by $\Delta t=0.5$. Each trajectory was generated by simulating Eq. (1) from a randomly perturbed initial condition and discarding an initial transient. The vorticity fields are all normalized, $\omega_{\text {train }}=\omega / \omega_{M}$, where $\omega_{M}=15$, which ensures $\left|\omega_{\text {train }}\right|<1$. Each vorticity field then has a random symmetry transform applied $\omega \rightarrow \mathscr{T}_{s} \mathscr{S}^{k} \mathscr{R}^{j} \omega$ to ensure the network sees the full state space.

The test data set used to generate the figures in this paper is constructed from a further 1000 trajectories in the same way.

\section{APPENDIX B: AUTOENCODER ARCHITECTURE}

The autoencoders discussed in this paper were all implemented using the Keras library [26]. All share a common structure consisting of a series of five convolutional layers with periodic padding. The number of filters (and kernel size) decreases sequentially, 128(8,8) $\rightarrow 64(8,8) \rightarrow$ $32(4,4) \rightarrow 16(4,4) \rightarrow 8(2,2)$. Nonlinear ReLU activation is applied to the output of each layer. Each convolution is followed by a max pooling operation over regions of size $2 \times 2$. 
(a)

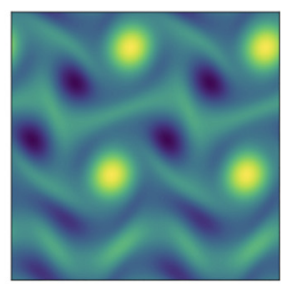

(e)

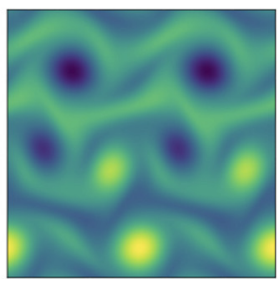

(b)

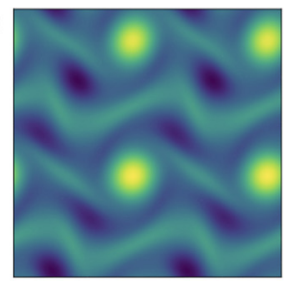

(f)

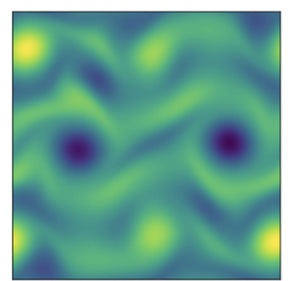

(c)

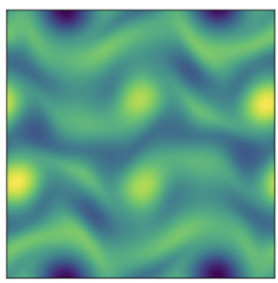

(g)

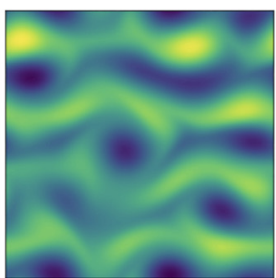

(d)

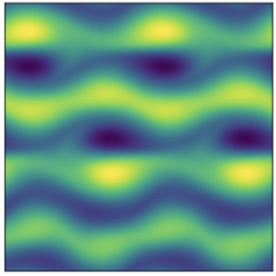

(h)

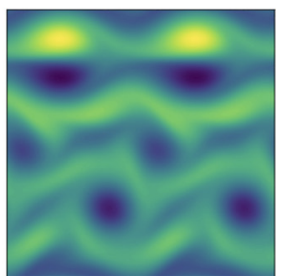

FIG. 9. Some bursting equilibria and traveling waves. (a)-(d) Vorticity field for four equilibria converged from projections and decodes onto $l=2$. The dissipation rate of these solutions is (a) $D / D_{l}=0.1974$, (b) $D / D_{l}=0.2042$, (c) $D / D_{l}=0.2233$, (d) $D / D_{l}=0.6058$. (e)-(h) Traveling waves. The phase speed and dissipation of these structures is (a) $c=0.0006, D / D_{l}=0.2184$; (b) $c=0.0053, D / D_{l}=0.2257$; (c) $c=$ $0.3178, D / D_{l}=0.4357$; (d) $c=0.1059, D / D_{l}=0.4536$.

The convolutions are followed by three fully connected layers, all with ReLU activation, which run $128 \rightarrow m \rightarrow 128$. The fully connected layers are followed by a series of five convolutional layers which mimics the encoder described above, with up sampling applied after each convolution on patches $2 \times 2$. A final convolutional layer with a tanh activation produces the output.

We trained networks with embedding dimension $m \in\{3,8,16,32,48,64,96,128\}$. Training was performed for 800 epochs for batch sizes of 64 and 128 using an Adam optimizer with a learning rate of 0.001 or 0.0003 . The results presented in this paper were generated using the best performing model at a given $m$. In order of increasing $m$, these hyper parameters are $(m$, learning rate, batch size) $(3,0.001,64),(8,0.001,128),(16,0.001,128),(32,0.001,128),(48,0.0003,64)$, $(64,0.0003,128),(96,0.0003,64),(128,0.0003,128)$.

\section{APPENDIX C: SIMPLE INVARIANT SOLUTIONS}

Equilibria and traveling waves correspond to solutions of Eq. (1), satisfying

$$
\mathscr{T}_{s=c T} \omega(\mathbf{x}, t+T)=\omega(\mathbf{x}, t) \quad \forall T,
$$

where the shift $s=c T$ is set by the fixed wave speed and $c=0$ for a pure equilibrium. The guesses take the form $\left(\omega_{g}, s_{g}\right)$, where we set the shift $s_{g}=c_{g} T_{\text {int }}=0$ and the integration time is held fixed at $T_{\text {int }}=3$. The vorticity guess is the decode of a projection onto the $l=2$ eigenspace [see Eq. (5) in the main text] from the embedding of a snapshot from within a 'bursting' episode.

The guesses are input into a Newton-Raphson algorithm which has been described extensively in previous research $[8,11,27]$. The size of the Jacobian matrix makes direct computation prohibitively expensive and updates to the solution $(\delta \omega, \delta s)$ are instead computed within a Krylov subspace (Newton-GMRES) which requires computation only of the action of the Jacobian on a vector. A hookstep is used to constrain updates of the guess to within a specified trust region [27].

Some example solutions converged from $l=2$ projections within bursting events are displayed in Fig. 9. Note the dipole structures seen in the simple invariant solutions with the highest dissipation values have not been seen in previously discovered exact coherent structures $[11,19]$. We have also converged a relative periodic orbit from within a bursting episode and have included snapshots from the evolution of this solution in Fig. 10. The flow field is dominated by four dipole structures; the 


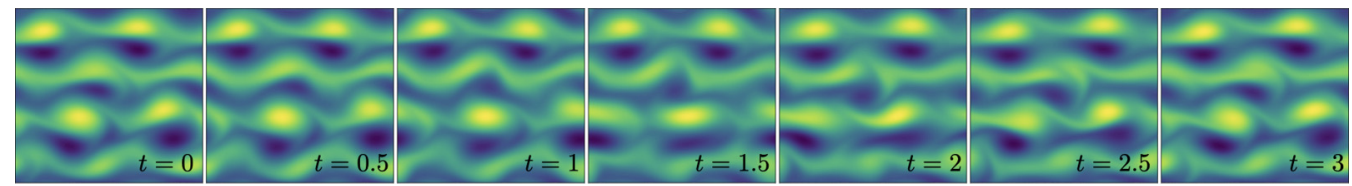

FIG. 10. Bursting periodic orbit. Snapshots separated by $\delta t=0.5$, labeled on each panel, from a discovered (relative) periodic orbit with period $T=3.41276$ and shift $s=-0.56022$.

lower pair propagate through the domain while the upper pair remain fixed in place. This bursting periodic orbit is qualitatively different from any documented previously [11,21].

[1] C. W. Rowley and S. T. M. Dawson, Model reduction for flow analysis and control, Ann. Rev. Fluid Mech. 49, 387 (2017).

[2] P. J. Schmid, Dynamic mode decomposition of numerical and experimental data, J. Fluid Mech. 656, 5 (2010).

[3] J. Page and R. R. Kerswell, Koopman mode expansions between simple invariant solutions, J. Fluid Mech. 879, 1 (2019).

[4] E. Hopf, A mathematical example displaying features of turbulence, Commun. Pure Appl. Math. 1, 303 (1948).

[5] G. Kawahara and S. Kida, Periodic motion embedded in plane Couette turbulence: Regeneration cycle and burst, J. Fluid Mech. 449, 291 (2001).

[6] R. R. Kerswell, Recent progress in understanding the transition to turbulence in a pipe, Nonlinearity $\mathbf{1 8}$, R17 (2005).

[7] B. Eckhardt, T. M. Schneider, B. Hof, and J. Westerweel, Turbulence transition in pipe flow, Annu. Rev. Fluid Mech. 39, 447 (2007).

[8] J. F. Gibson, J. Halcrow, and P. Cvitanovic, Visualizing the geometry of state space in plane Couette flow, J. Fluid Mech. 611, 107 (2008).

[9] G. Kawahara, M. Uhlmann, and L. van Veen, The significance of simple invariant solutions in turbulent flows, Annu. Rev. Fluid Mech. 44, 203 (2012).

[10] P. Cvitanović, R. Artuso, R. Mainieri, G. Tanner, and G. Vattay, Chaos: Classical and Quantum (Niels Bohr Inst., Copenhagen, 2016).

[11] G. J. Chandler and R. R. Kerswell, Invariant recurrent solutions embedded in a turbulent two-dimensional Kolmogorov flow, J. Fluid Mech. 722, 554 (2013).

[12] Y. LeCun, Y. Bengio, and G. Hinton, Deep learning, Nature 521, 436 (2015).

[13] V. Gulshan, L. Peng, M. Coram, M. C. Stumpe, D. Wu, A. Narayanaswamy, S. Venugopalan, K. Widner, T. Madams, J. Cuadros, R. Kim, R. Raman, P. Q. Nelson, J. Mega, and D. Webster, Development and validation of a deep learning algorithm for detection of diabetic retinopathy in retinal fundus photographs, JAMA 316, 2402 (2016).

[14] J. Pathak, B. Hunt, M. Girvan, Z. Lu, and E. Ott, Model-Free Prediction of Large Spatiotemporally Chaotic Systems from Data: A Reservoir Computing Approach, Phys. Rev. Lett. 120, 024102 (2018).

[15] A. J. Linot and M. D. Graham, Deep learning to discover and predict dynamics on an inertial manifold, Phys. Rev. E 101, 062209 (2020).

[16] Y. Bar-Sinai, S. Hoyer, J. Hickey, and M. P. Brenner, Learning data-driven discretizations for partial differential equations, Proc. Natl. Acad. Sci. 116, 15344 (2019).

[17] V. I. Arnol'd and L. D. Meshalkin, The seminar of A.N. Kolmogorov on selected topics in analysis, Usp. Mat. Nauk 15, 247 (1960).

[18] N. Platt, L. Sirovich, and N. Fitzmaurice, An investigation of chaotic Kolmogorov flows, Phys. Fluids 3, 681 (1991). 
[19] M. Farazmand, An adjoint-based approach for finding invariant solutions of Navier-Stokes equations, J. Fluid Mech. 795, 278 (2016).

[20] C. W. Rowley, I. Mezić, S. Bagheri, P. Schlatter, and D. S. Henningson, Spectral analysis of nonlinear flows, J. Fluid Mech. 641, 115 (2009).

[21] D. Lucas and R. Kerswell, Spatiotemporal dynamics in two-dimensional Kolmogorov flow over large domains, J. Fluid Mech. 750, 518 (2014).

[22] L. van der Maaten and G. E. Hinton, Visualizing high-dimensional data using t-SNE, J. Machine Learning Res. 9, 2579 (2008).

[23] J. Page, R. R. Kerswell, and M. P. Brenner, Searching for periodic orbits in turbulent flows using machine learning, 71st Annual Meeting of the APS Division of Fluid Dynamics, APS Bulletin, Vol. 63 (American Physical Society, 2018), Abstract No.BAPS.2018.DFD.D01.6.

[24] R. Artuso, E. Aurell, and P. Cvitanovic, Recycling of strange sets: I. Cycle expansions, Nonlinearity 3, 325 (1990).

[25] R. Artuso, E. Aurell, and P. Cvitanovic, Recycling of strange sets: II. Applications, Nonlinearity 3, 361 (1990).

[26] F. Chollet, Keras, https://github.com/fchollet/keras (2015).

[27] D. Viswanath, Recurrent motions within plane Couette turbulence, J. Fluid Mech. 580, 339 (2007). 